Original paper

\title{
IgG4 subclass and gamma-glutamyl transferase in children with ulcerative colitis with primary sclerosing cholangitis and without sclerosing cholangitis
}

\author{
Fatemeh Farahmand ${ }^{1}$, Mitra Ahmadi ${ }^{1}$, Ahmad Khodadad' ${ }^{1}$, Mehri Najafi', Gholamhossein Fallahi', Farzaneh Motamed', \\ Masoud Movahedi', Asghar Aghamohammadi², Nima Rezaei ${ }^{2,3,4}$, Hazhir Javaherizadeh ${ }^{5}$ \\ 'Department of Pediatric Gastroenterology, Pediatrics Center of Excellence, Children's Medical Center, Tehran University of Medical Sciences, Tehran, Iran \\ ${ }^{2}$ Research Center for Immunodeficiencies, Children's Medical Center, Tehran University of Medical Sciences, Tehran, Iran \\ ${ }^{3}$ Department of Immunology, School of Medicine; and Molecular Immunology Research Center, Tehran University of Medical Sciences, Tehran, Iran \\ ${ }^{4}$ Universal Scientific Education and Research Network (USERN), Tehran, Iran \\ ${ }^{5}$ Alimentary Tract Research Center, Ahvaz Jundishapur University of Medical Sciences, Ahvaz, Iran
}

\begin{abstract}
Aim of the study: Primary sclerosing cholangitis (PSC) is a chronic cholestatic liver disease which could be associated with inflammatory bowel disease (IBD), particularly ulcerative colitis (UC). The aim of this study was to compare GGT and lgG4 levels among children with UC with PSC and without PSC.

Material and methods: In this cross sectional study children with UC with PSC and UC without PSC were included. Serum immunoglobulin G4 (IgG4) and gamma-glutamyl transpeptidase (GGT) levels of the 90 UC patients with and without concomitant PSC were measured. Children with serum lgG4 concentration $>175 \mathrm{mg} / \mathrm{dl}$ were considered to have elevated lgG4.

Results: Elevated serum lgG4 was found in 8 of $30(26.6 \%)$ patients with PSC vs. 3 of $60(5.0 \%)$ patients without PSC. Compared with the group without symptoms of PSC, the group with PSC showed significantly higher levels of aspartate aminotransferases (AST; $22.5 \mathrm{U} / \mathrm{l}$ vs. $70.0 \mathrm{U} / \mathrm{l}, p<0.001$ ), alkaline phosphatase (ALP; $359.0 \mathrm{U} / \mathrm{I}$ vs. $602.0 \mathrm{U} / \mathrm{l}, p<0.001$ ), and $\operatorname{lgG} 4(56.0 \mathrm{vs}$. 73.0, $p=0.02$ ). The odd ratio of the elevated IgG4 and GGT in predicting PSC was 6.9 (95\% Cl: 1.6-28.4) and 18 (95\% Cl: 5.7-55.9), respectively.

Conclusions: AST, alanine aminotransferase (ALT), GGT, ALP, and serum IgG4 were significantly higher in UC patients with sclerosing cholangitis (SC) compared to UC patients without SC. GGT and IgG-4 measurements are recommended for evaluation of UC.
\end{abstract}

Key words: primary sclerosing cholangitis, inflammatory bowel disease, ulcerative colitis, lgG4.

\section{Address for correspondence}

Mitra Ahmadi, MD, Children's Medical Center Hospital, 62 Qarib St, Keshavarz Blvd, Tehran 14194, Iran, e-mail: mitra_ahm92@yahoo.com

\section{Introduction}

Ulcerative colitis (UC) is an inflammatory bowel disease that affects quality of life of the patients [1]. Primary sclerosing cholangitis (PSC) is a chronic cholestatic liver disease characterized by progressive destruction of both intrahepatic and extrahepatic bile ducts and eventual development of biliary cirrhosis $[2,3]$. The etiology of this condition is poorly under- stood, but there is some evidence suggesting that autoimmune phenomena play a role in the disease pathogenesis $[4,5]$. The majority of patients with PSC have underlying inflammatory bowel disease (IBD), i.e. UC or Crohn's disease $[6,7]$. Patients with PSC are more likely to have UC than Crohn's disease (90\% vs. $10 \%$ ), with approximately $5-8 \%$ of all UC patients having PSC [8-10]. Primary sclerosing cholangitis is relatively infrequent in children, with a likely incidence less than 
$20 \%$ of that reported for adults [11]. Despite this, PSC remains an important cause of morbidity and mortality in children and accounted for approximately $2 \%$ of the liver transplants performed in this group of patients [11].

The recent recognition of immunoglobulin G4-related sclerosing cholangitis (IgG4-SC) found in association with autoimmune pancreatitis has led to the recognition that some previously diagnosed cases of PSC were in fact IgG4-related diseases [12-14]. Findings show that serum IgG4 levels are often elevated in PSC, and IgG4 plasma cells are frequent in PSC liver explants [15]. In addition, the IgG4-related diseases are typically responsive to corticosteroids, which are not seen in PSC [13]. In this regard, measuring the IgG4 and gamma-glutamyl transpeptidase (GGT) level in patients with PSC to explore a possible common pathway in the pathogenesis of these diseases seems to be crucial and can provide a treatment protocol based on corticosteroid for these patients. Considering the above information, we measured IgG4 concentration in a group of UC patients with and without PSC, and evaluated its predicting effects for PSC.

\section{Material and methods}

\section{Study population}

This comparative cross-sectional study was conducted from January 2014 to May 2015, in the Children's Medical Center in Tehran, Iran. The center functions both as a primary and as a referral center for children with severe liver disorders. A total of

Table 1. Comparison of demographic, clinical, and laboratory data between ulcerative colitis patients with and without primary sclerosing cholangitis

\begin{tabular}{lccc}
\hline Variable & Cases (30) & Controls (60) & P-value \\
\hline Age (years) & $11.1(4.13)$ & $11.3(3.45)$ & 0.80 \\
\hline $\begin{array}{l}\text { Age at diagnosis of UC } \\
\text { (years) }\end{array}$ & $8.2(3.71)$ & $9.2(3.20)$ & 0.172 \\
\hline $\begin{array}{l}\text { Age at diagnosis of PSC } \\
\text { (years) }\end{array}$ & $9.96(3.45)$ & $\mathrm{NA}$ & $\mathrm{NA}$ \\
\hline AST (unit /l) & $70.0(58.75)$ & $22.5(21.75)$ & 0.001 \\
\hline ALT(unit/l) & $70.0(61)$ & $21.5(24.75)$ & 0.001 \\
\hline ALP (IU/l) & $602.0(676.75)$ & $359.0(301)$ & 0.001 \\
\hline GGT (IU/l) & $111.0(211.5)$ & $21.5(17.75)$ & 0.001 \\
\hline Total lgG (mg/dl) & $1534.0(890.5)$ & $950.0(427)$ & 0.001 \\
\hline lgG4 (mg/dl) & $73.0(266.5)$ & $56.0(39.5)$ & 0.02 \\
\hline
\end{tabular}

UC - ulcerative colitis, PSC - primary sclerosing cholangitis, AST - aspartate aminotransferases, ALT - alanine aminotransferase, ALP - alkaline phosphatase, GGT - gamma-glutamyl transpeptidase, NA - not available
90 children with UC (with PSC and without PSC) were included in the study. The diagnosis of PSC was based on accepted criteria including clinical, biochemical, and radiological findings [16]. After observation of a clinical or biochemical abnormality, patients were diagnosed with sclerosing cholangitis (SC) if segmental bile duct stricture was documented by magnetic resonance cholangiopancreatography (MRCP). Patients with autoimmune diseases (systemic lupus erythematosus, vasculitis) and allergic disorders (asthma, atopic dermatitis) were excluded from further analysis. Standard clinical data including demographic, clinical, laboratory and radiological features of patients with PSC were documented. All these patients had previously given informed consent for use of stored sera, and for review of medical records.

\section{Immunoglobulin G4 determination}

Immunoglobulin G4 was measured at least once in all patients attending the clinic with a diagnosis of PSC. In our study, patients with serum IgG4 concentration $>175 \mathrm{mg} / \mathrm{dl}$ were considered to have elevated IgG4 [17]. The method used to measure serum IgG4 concentration was immunonephelometry (MININEPH Nephelometer, the Binding Site Ltd. USA).

\section{Statistical analysis}

Statistical analysis was performed using the chisquare test and nonparametric Mann-Whitney $U$ test to compare the variables. $P$-values less than 0.05 were considered statistically significant. Data were analyzed using SPSS version 18 software.

This study was approved by the ethical committee of the hospital.

\section{Results}

During the study period, of the 90 patients who were diagnosed with UC, 30 (33.3\%) had PSC (cases) and 60 (66.6\%) had no symptoms of PSC (controls). The mean age of case and control groups was $13.1 \pm 4.1$ and $11.3 \pm 3.4$ years, respectively. Forty-four $(48.8 \%)$ patients with UC were girls and $46(51.2 \%)$ were boys $(p>0.05)$. Serum IgG4 levels were elevated in $8(26.6 \%)$ of the patients with PSC, whereas 3 patient without PSC (5.0\%) had elevated serum IgG4.

Table 1 shows the comparison between the two UC groups (with and without PSC) in regards to age, age at diagnosis of UC, aspartate aminotransferases (AST) level, alkaline phosphatase (ALP) and gamma-glutamyl transpeptidase (GGT) levels and IgG4 levels. Com- 
pared with the group without symptoms of PSC, the group with PSC (cases) showed significantly higher levels of AST (22.5 U/l vs. $70.0 \mathrm{U} / 1, p<0.001$ ), ALP (359.0 U/l vs. $602.0 \mathrm{U} / 1, p<0.001)$, and IgG4 (56.0 vs. $73.0, p=0.02)$.

The odds ratio of elevated IgG4 and GGT in predicting PSC was 6.9 (95\% CI: 1.6-28.4) and 18 (95\% CI: 5.7-55.9), respectively (Table 2).

Measuring the mean duration (years) of UC indicated that there was a significant difference in the duration of the disease between patients with and without PSC with a median duration of 2 vs. 1 years $(p=0.02)$, respectively.

The mean disease duration and the mean age at diagnosis in the group with normal serum IgG4 and the group with elevated IgG4 showed no significant difference, $p$ value $>0.05$ (Table 3 ).

\section{Discussion}

In the present study, we analyzed the role of serum IgG4 levels in the diagnosis of immune-related SC in the patients with UC. According to our results, IgG4 levels were elevated in 8 of $30(26.6 \%)$ patients with PSC, whereas only three patients without symptoms of PSC (5.0\%) had elevated serum IgG4. This rate of patients with elevated serum IgG4 was comparable with those of previous studies from Iran. During 2011-2012, Parhizkar and others reported that 9 of 34 (26.5\%) patients with diagnosed UC had elevated serum concentrations of IgG4 [18]. In this survey, we also measured the odd ratio of elevated IgG4 in predicting PSC. The presence of high levels of serum IgG4 was significantly associated with a greater than 7-fold higher risk of PSC (Table 3). This finding suggests that IgG4 measurement should be considered in all patients with PSC.

In the study by Lian et al., elevated serum IgG4 $\geq 1.25$ upper limit of normal range (ULN) shows excellent predictability to distinguish IgG4-SC among SC patients [19]. In the study by Taghavi et al., high serum IgG-4 was detected in a higher percentage of patients with PSC [20].

Moreover, several other important factors such as AST, ALT and ALP score were significantly higher in patients with PSC than those in control subjects, which seem to be valuable criteria in PSC diagnosis. Other parameters of the patients (MRCP, calprotectin and P-ANCA) were also pronounced; however, statistical significance was not achieved, in part probably because of unavailable laboratory data or liver biopsy specimens. In the previous study, several variables such as laboratory and imaging findings, presence of IBD, esophageal varices, ascites, age, smoking and
Table 2. Odds ratio of elevated immunoglobulin G4 (IgG4) and gammaglutamyl transpeptidase (GGT) in predicting primary sclerosing cholangitis

\begin{tabular}{lcccc}
\hline & $\begin{array}{c}\text { Crude OR } \\
(95 \% \mathrm{Cl})\end{array}$ & $\boldsymbol{P}$-value & $\begin{array}{c}\text { Adjusted } \\
\text { OR }(95 \% \mathrm{Cl})\end{array}$ & $P$-value \\
\hline IgG4 & $\begin{array}{c}6.9 \\
(1.67-28.44)\end{array}$ & 0.007 & $\begin{array}{c}7.44 \\
(1.74-31.72)\end{array}$ & 0.007 \\
\hline GGT & $\begin{array}{c}18 \\
(5.78-55.98)\end{array}$ & 0.001 & $\begin{array}{c}18.17 \\
(5.78-57.07)\end{array}$ & 0.007 \\
& & & & \\
\hline
\end{tabular}

OR - odd ratio

Table 3. Mean disease duration and the mean age at diagnosis in the studied groups

\begin{tabular}{lccc}
\hline & $\begin{array}{c}\text { Cases with } \\
\text { elevated IgG4 }\end{array}$ & $\begin{array}{c}\text { Cases without } \\
\text { elevated lgG4 }\end{array}$ & P-value \\
\hline Mean disease duration (years) & 2 & 2.5 & 0.9 \\
\hline Mean age at diagnosis (years) & 8.5 & 9 & 0.7 \\
\hline
\end{tabular}

Child score were analyzed in patients with elevated IgG4 and compared with patients with normal IgG4 concentration [18]. According to their results, they did not find any significant relationship among patients with and without elevated IgG4 in terms of investigated parameters [18]. In the Mendes et al. study, 12 of 127 (9.4\%) patients with increased IgG4 levels had higher risk scores and less time for liver transplantation [2].

In the current study, GGT level was significantly higher in UC patients with SC than patients without SC. In the study by Goyal et al., elevated GGT and ALT in patients with UC was associated with markedly increased UC associated liver disease [21]. In the recent study by Deneau et al., GGT normalization or reduction was associated with a favorable outcome among UC children with PSC [22].

Some limitations of this study should be considered for interpretation of results. First, although we started with a large number of patients, after several screenings, the number of eligible patients was quite small. This may be due to the fact that the prevalence of PSC in children is less than $20 \%$ of that reported for adults. Second, the potential influence of some important factors could not be analyzed because of the limited information obtained from the studied patients. Due to financial limitations, only IgG-4 and GGT were used in this study.

In conclusion, relatively high levels of serum IgG4 in patients with UC and PSC were seen. In this regard, measuring IgG4 and GGT in PSC patients may be considered and should be a part of the diagnostic criteria in these kinds of patients. Further studies to clarify the true nature of IgG4-related SC and its response to corticosteroid therapy and other useful therapies are needed. 


\section{Disclosure}

The authors report no conflict of interest.

\section{References}

1. Kawalec P, Stawowczyk E, Mossakowska M, et al. Disease activity, quality of life, and indirect costs of ulcerative colitis in Poland. Prz Gastroenterol 2017; 12: 60-65.

2. Mendes FD, Jorgensen R, Keach J, et al. Elevated serum IgG4 concentration in patients with primary sclerosing cholangitis. Am J Gastroenterol 2006; 101: 2070-2075.

3. Nakanuma Y, Harada K, Katayanagi K, et al. Definition and pathology of primary sclerosing cholangitis. J Hepatobiliary Pancreat Surg 1999; 6: 333-342.

4. Worthington J, Cullen S, Chapman R. Immunopathogenesis of primary sclerosing cholangitis. Clin Rev Allergy Immunol 2005; 28: 93-103.

5. Aoki CA, Bowlus CL, Gershwin ME. The immunobiology of primary sclerosing cholangitis. Autoimmun Rev 2005; 4: 137 143.

6. Loftus EV Jr., Harewood GC, Loftus CG, et al. PSC-IBD: a unique form of inflammatory bowel disease associated with primary sclerosing cholangitis. Gut 2005; 54: 91-96.

7. O’Toole A, Alakkari A, Keegan D, et al. Primary sclerosing cholangitis and disease distribution in inflammatory bowel disease. Clin Gastroenterol Hepatol 2012; 10: 439-441.

8. Loftus EV, Jr. Clinical epidemiology of inflammatory bowel disease: Incidence, prevalence, and environmental influences. Gastroenterology 2004; 126: 1504-1517.

9. Alreheili KM, Alsaleem KA, Almehaidib AI. Natural history and outcome of inflammatory bowel diseases in children in Saudi Arabia: A single-center experience. Saudi J Gastroenterol 2018; 24 : 171-176.

10. Fagundes EDT, Ferreira AR, Hosken CC, et al. Primary sclerosing cholangitis in children and adolescents. Arq Gastroenterol 2017; 54: 286-291.

11. Shneider BL. Diagnostic and therapeutic challenges in pediatric primary sclerosing cholangitis. Liver Transpl 2012; 18: 277-281.

12. Nakazawa T, Naitoh I, Hayashi K, et al. Diagnostic criteria for IgG4-related sclerosing cholangitis based on cholangiographic classification. J Gastroenterol 2012; 47: 79-87.

13. Ohara H, Okazaki K, Tsubouchi $\mathrm{H}$, et al. Clinical diagnostic criteria of IgG4-related sclerosing cholangitis 2012. J Hepatobiliary Pancreat Sci 2012; 19: 536-542.

14. Ohara H, Nakazawa T, Kawa S, et al. Establishment of a serum IgG4 cut-off value for the differential diagnosis of IgG4-related sclerosing cholangitis: a Japanese cohort. J Gastroenterol Hepatol 2013; 28: 1247-1251.

15. Webster GJ, Pereira SP, Chapman RW. Autoimmune pancreatitis/IgG4-associated cholangitis and primary sclerosing cholangitis - overlapping or separate diseases? J Hepatol 2009; 51: 398-402.

16. Chapman R, Fevery J, Kalloo A, et al. Diagnosis and management of primary sclerosing cholangitis. Hepatology 2010; 51: 660-678.

17. Kamisawa T, Okamoto A. IgG4-related sclerosing disease. World J Gastroenterol 2008; 14: 3948-3955.

18. Parhizkar B, Mohammad Alizadeh AH, Asadzadeh Aghdaee H, et al. Primary sclerosing cholangitis associated with elevated immunoglobulin-g4: a preliminary study. ISRN Gastroenterol 2012; 2012: 325743 .
19. Lian M, Li B, Xiao X, et al. Comparative clinical characteristics and natural history of three variants of sclerosing cholangitis: IgG4-related SC, PSC/AIH and PSC alone. Autoimmun Rev 2017; 16: 875-882.

20. Taghavi SA, Majd SK, Sianati M, et al. Prevalence of IgG-4-associated cholangiopathy based on serum IgG-4 levels in patients with primary sclerosing cholangitis and its relationship with inflammatory bowel disease. Turk J Gastroenterol 2016; 27: 547-552.

21. Goyal A, Hyams JS, Lerer T, et al. Liver enzyme elevations within 3 months of diagnosis of inflammatory bowel disease and likelihood of liver disease. J Pediatr Gastroenterol Nutr 2014; 59: 321-323.

22. Deneau MR, Mack C, Abdou R, et al. Gamma glutamyltransferase reduction is associated with favorable outcomes in pediatric primary sclerosing cholangitis. Hepatol Commun 2018; 2: 1369-1378. 